\title{
Removal of SARS-CoV-2 bioaerosols using ultraviolet air filtration
}

\author{
Roy E. Barnewall DVM, $\mathrm{PhD}^{1}$ and Werner E. Bischoff MD, $\mathrm{PhD}^{2}$ (1) \\ ${ }^{1}$ Life Sciences Research, Battelle Biomedical Research Center, Columbus, Ohio and ${ }^{2}$ Infection Prevention and Health System Epidemiology, Wake Forest Baptist \\ Health, Winston-Salem, North Carolina
}

\begin{abstract}
Engineering controls play an important role in reducing the spread of severe acute respiratory coronavirus virus 2 (SARS-CoV-2). ${ }^{1}$ Established technologies such as air filtration, and novel approaches such as ultraviolet (UV)-C light or plasma air ionization, have the potential to support the fight against the coronavirus disease 2019 (COVID-19) pandemic. ${ }^{2}$ We tested the efficacy of an air purification system (APS) combining UV-C light and high-efficiency particulate air (HEPA) filtration in a controlled environment using SARS-CoV-2 as test organism. The APS successfully removed the virus from the air using UV-C light by itself and in combination with HEPA air filtration.
\end{abstract}

\section{Methods}

Testing was performed at the Battelle Biomedical Research Center (Columbus, Ohio) using 3 test groups: (1) inactive test unit (control); (2) APS with UV-C light; (3) APS with UV-C light and HEPA filter in place. The 10-minute test runs in each group were repeated 5 times.

The APS consisted of a UV-C photolytic chamber, which incorporated 4 low-pressure UV-C germicidal lamps operating at the 254-nm wavelength at a total irradiance of $177.8 \mu \mathrm{w} / \mathrm{cm}^{2}$ and containing 2,400 transparent quartz tubular elements oriented randomly in the chamber (Aerobiotix, Dayton, OH). Air filtration was provided by a commercial-grade $99.97 \%$ efficacy HEPA filter (Aerobiotix, Dayton, Ohio).

The APS was placed inside a single-pass air-testing unit (ATU; 30$\mathrm{cm} \times 30$-cm [12-inch $\times 12$-inch] cross section) within a biological safety level 3 cabinet (Fig. 1). The ATU consisted of a pre-APS chamber, an APS chamber, and a post-APS chamber. Airflow was controlled by fans in the ATU, and recirculation occurred at 588-623 L per minute. A 6-jet Collison nebulizer (BGI, Waltham, MA) generated a SARS-CoV-2 aerosol from liquid suspension using HEPA and carbon capsule prefiltered air (Pall, Port Washington, NY). In total, a $1.57 \times 10^{7} 50 \%$ tissue-culture infective dose $\left(\mathrm{TCID}_{50}\right)$ of SARS-CoV-2 was nebulized into the pre-APS chamber at $0.4 \mathrm{~mL}$ per minute for 10 minutes and was mixed by baffles inside the chamber. The mixing air volume was $567 \mathrm{~L}$ per minute. This setup represents a pre-APS chamber air concentration of 2,760 units/L, assuming full dissemination efficiency. An air-particle sizer (APS model 3321 with aerosol diluter model 3302A; TSI, Shoreview, MN) measured the aerodynamic particles at $0.25 \mathrm{~L} /$ minute for 10 seconds at 5 minutes into the control run. Impinger samples (model 7541, Ace Glass) were taken in the pre- and post-APS chambers during the control runs at $6.0 \pm 0.5 \mathrm{~L}$ per minute for 10 minutes each and from the post-APS chamber for all

Author for correspondence: Werner Bischoff, E-mail: wbischof@wakehealth.edu Cite this article: Barnewall RE and Bischoff WE. (2021). Removal of SARS-CoV-2 bioaerosols using ultraviolet air filtration. Infection Control \& Hospital Epidemiology, 42: 1014-1015, https://doi.org/10.1017/ice.2021.103 subsequent runs. Temperature and humidity during all tests were $68-69^{\circ} \mathrm{F}$ and $48 \%-49 \%$ relative humidity, respectively.

The SARS-CoV-2 virus was propagated by the American Type Culture Collection (ATCC) from BEi Resources NR-52281 lot 70034262 (ATCC, Manassas, VA) and concentrated to $1.24 \times 10^{7}$ $\mathrm{TCID}_{50} / \mathrm{mL}$ in minimum essential medium (Sigma cat. no. 51 416C; Sigma-Aldrich, St Louis, MO) + penicillin-streptomycin (Sigma cat. no. P4333;) $+5 \%$ fetal bovine serum (Sigma cat. no. F4135).

Samples were quantitated using VERO E6 cell culture (ATCC C1008 E6 cat. no. NR596) to determine cytopathological effects (CPEs), which included cell rounding, fusion, or lysis and were expressed as present or absent in the cell culture. The analysis of these results was performed using the Fisher-Boschloo test.

\section{Results}

In the control group, particle counts increased from 20 (in the pre-APS chamber) to 54 particles (in the post-APS chamber) after the introduction of the SARS-CoV-2 aerosol. A similar yet less pronounced increase was observed in the second run. In the APS with UV-C light, particle counts increased from 79 in the pre-APS chamber to 101 in the post-APS chamber. Placing a HEPA filter into the airflow reduced the particle count significantly, from 79 to 5 particles. The mass mean aerodynamic diameter over all runs ranged from 0.80 to $1.17 \mu \mathrm{m}$, consistent with the Collison nebulizer particle dispersion characteristics. ${ }^{3}$

For the viral culture tests, 3 of 5 runs collected from the pre-APS chamber samples and 2 of 5 runs collected in the post-APS chamber showed CPEs in the control group. No CPE was observed in the post-APS chamber samples for either the APS with UV-C light activated or the APS with UV-C light and HEPA filtration. The 1 -sided Fisher-Boschloo test revealed a $P$ value of .0386 .

In conclusion, the emergence of SARS-CoV-2 has accelerated the development and adoption of air purification technologies. ${ }^{4-7}$ In this study, viable virus was detected in the control runs without UV-C light activation or HEPA filtration. Using an UV-C light system with and without HEPA filtration eliminated SARS-CoV-2.

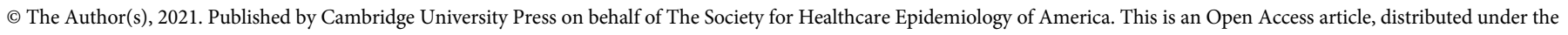

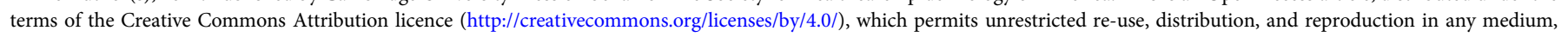
provided the original work is properly cited. 


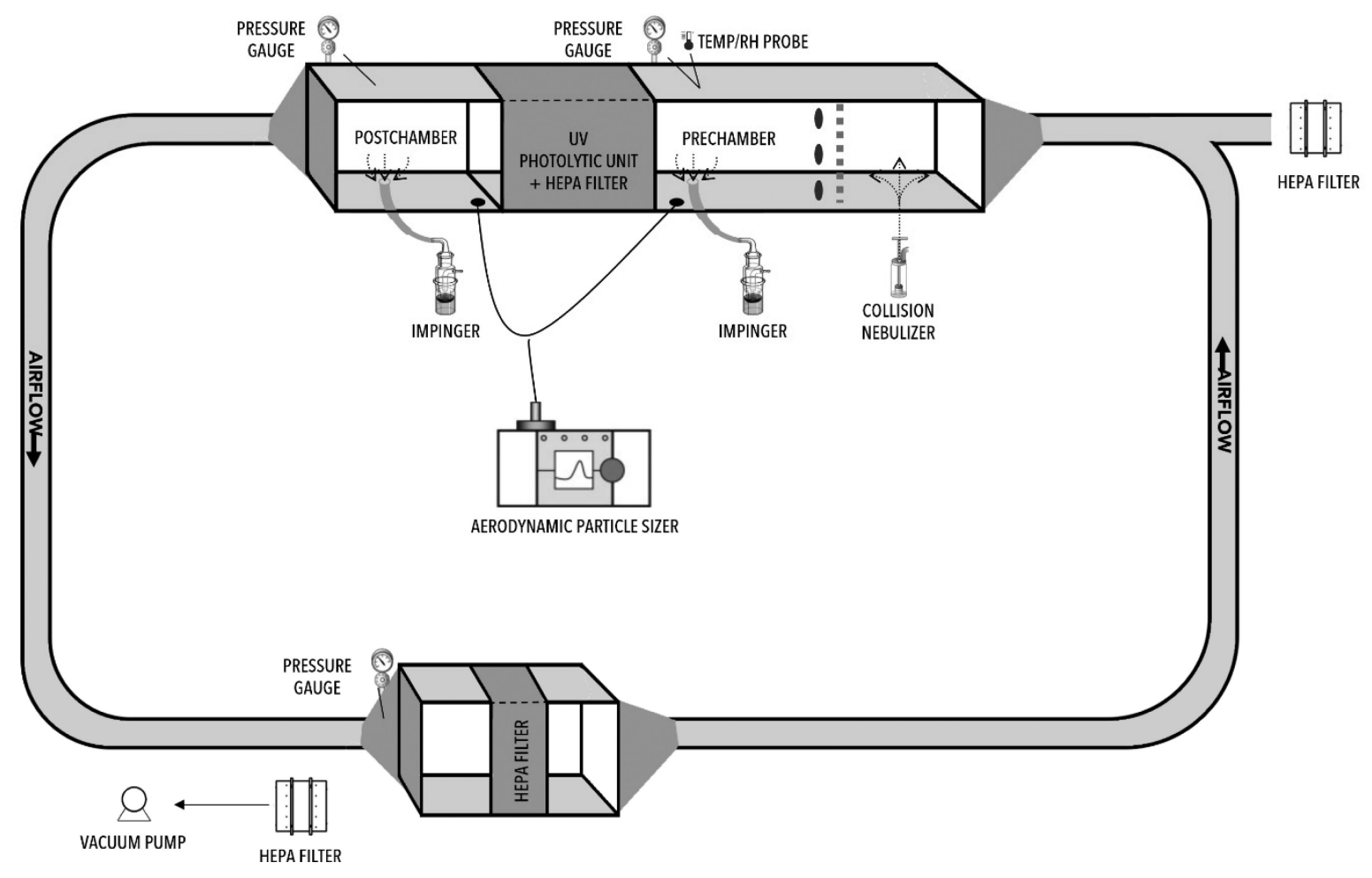

Fig. 1. Schematic of testing setup.

This study has several key advantages. Virus was detected by direct culturing, which depicts the presence of viable virus, unlike genomic studies. The closed single-pass design assured that bioaerosols were contained in the sealed test unit, reducing environmental effects such as decreased viability of the aerosolized virus through temperature and humidity and adherence to chamber walls. ${ }^{8}$ Airflow was maximized to challenge the APS, which resulted in a SARS-CoV-2 preAPS chamber air concentration of 2,760 $\mathrm{TCID}_{50}$ units/L, assuming full dissemination efficiency. Even accounting for inefficiencies, the pre-APS chamber air concentration compared favorably to published SARS-CoV-2 air concentrations in samples from active COVID-19 patient rooms $\left(6-74 \mathrm{TCID}_{50}\right.$ units/L). ${ }^{9}$

This study has several limitations. Maximizing airflow resulted in rapid dilution of the aerosolized virus. Therefore, although the presence of viable virus through $\mathrm{CPE}$ could be detected, quantification could not be performed. However, these findings were significant and are consistent with prior testing of the same device using MS2 virus. ${ }^{10}$

To date, we are unaware of any APS having been directly challenged with SARS-CoV-2 bioaerosols. ${ }^{4-7}$ The APS successfully eliminated the virus within closely defined parameters reaching significance. Future studies should address the impact of APS on SARS-CoV-2 transmission in a clinical environment, considering challenges from virus emissions by patients to airflow patterns.

Acknowledgments.

Financial support. This study was supported by Aerobiotix, Dayton, Ohio.

Conflicts of interest. Dr Barnewall reports grants from Aerobiotix (Dayton, $\mathrm{OH}$ ) during the conduct of the study. Dr Bischoff reports grants from Aerobiotix outside the submitted work.

\section{References}

1. Guidance on preparing workplaces for COVID-19. Occupational Safety and Health Administration website. https://www.osha.gov/Publications/ OSHA3990.pdf. Published 2020. Accessed November 18, 2020.

2. Morawska L, Tang JW, Bahnfleth W, et al. How can airborne transmission of COVID-19 indoors be minimised? Environ Int 2020;142:105832.

3. Thomas RJ, Webber D, Sellors W, et al. Characterization and deposition of respirable large- and small-particle bioaerosols. Appl Environ Microbiol 2008;74:6437-6443.

4. Qiao Y, Yang M, Marabella IA, et al. Greater than 3-log reduction in viable coronavirus aerosol concentration in ducted ultraviolet-C (UV-C) systems. Environ Sci Technol 2020. doi: 10.1021/acs.est.0c05763.

5. Heßling M, Hönes K, Vatter P, Lingenfelder C. Ultraviolet irradiation doses for coronavirus inactivation-review and analysis of coronavirus photoinactivation studies. GMS Hyg Infect Control 2020 May 14. doi: 10.3205/ dgkh000343.

6. Buonanno M, Welch D, Shuryak I, Brenner DJ. Far-UVC light (222 nm) efficiently and safely inactivates airborne human coronaviruses. Sci Rep 2020;10:10285.

7. Anghel L, Popovici CG, Stătescu C, et al. Impact of HVAC-systems on the dispersion of infectious aerosols in a cardiac intensive care unit. Int $J$ Environ Res Public Health 2020;17:6582.

8. Bedrosian N, Mitchell E, Rohm E, et al. A systematic review of surface contamination, stability, and disinfection data on SARS-CoV-2 (through July 10, 2020). Environ Sci Technol 2020. doi: 10.1021/acs.est.0c05651.

9. Lednicky JA, Lauzardo M, Fan ZH, et al. Viable SARS-CoV-2 in the air of a hospital room with COVID-19 patients. Int $J$ Infect Dis 2020;100:476-482.

10. Aerobiotix White Paper. Elimination of aerosolized virus in single-pass testing using aerobiotix ultraviolet air handling system. Aerobiotix website. https://aerobiotix.com/project/elimination-of-aerosolized-virus-in-singlepass-testing-2/ Published 2013. Accessed January 6, 2021. 